\title{
Ten tips for developing interdisciplinary socio-ecological researchers
}

\author{
Rachel Kelly 1,2 (1) Mary Mackay $y^{1,3} \cdot$ Kirsty L. Nash ${ }^{1,2} \cdot$ Christopher Cvitanovic $^{1,4} \cdot$ Edward H. Allison $^{5}$. \\ Derek Armitage $^{6} \cdot$ Aletta Bonn $^{7,8,9} \cdot$ Steven J. Cooke $^{10} \cdot$ Stewart Frusher $^{1,2} \cdot$ Elizabeth A. Fulton $^{1,11}$. \\ Benjamin S. Halpern ${ }^{12,13}$. Priscila F. M. Lopes ${ }^{14}$ - E. J. Milner-Gulland ${ }^{15} \cdot$ Myron A. Peck $^{16}$. Gretta T. Pecl ${ }^{1,2}$. \\ Robert L. Stephenson ${ }^{17,18} \cdot$ Francisco Werner $^{19}$
}

Received: 31 January 2019 / Accepted: 10 June 2019 / Published online: 12 July 2019

(c) Springer Nature Singapore Pte Ltd. 2019

\begin{abstract}
Interdisciplinary research and collaborations are essential to disentangle complex and wicked global socio-ecological challenges. However, institutional structures and practices to support interdisciplinary research are still developing and a shared understanding on how best to develop effective interdisciplinary researchers (particularly at early career stages) is lacking. Barriers to interdisciplinary approaches, which include diverse disciplinary 'languages', research time constraints and limited guidance on how to achieve interdisciplinarity in practice, further challenge this understanding. To help overcome these barriers, this paper provides practical advice for early career researchers and their mentors, as well as senior researchers and lab leaders, in the form of 10 tips: 'Develop an area of expertise'; 'Learn new languages'; 'Be open-minded'; 'Be patient'; 'Embrace complexity'; 'Collaborate widely; 'Push your boundaries'; 'Consider if you will engage in interdisciplinary research'; 'Foster interdisciplinary culture'; and 'Champion interdisciplinary researchers'. They are presented here to empower present and future generations of interdisciplinary researchers in their endeavour to solve contemporary socioecological challenges worldwide.
\end{abstract}

Keywords Collaborative research $\cdot$ Early career researcher $\cdot$ Interdisciplinary research (IDR) $\cdot$ Socio-ecological challenges

Rachel Kelly

r.kelly@utas.edu.au

1 Centre for Marine Socioecology, University of Tasmania, Hobart, TAS 7005, Australia

2 Institute for Marine and Antarctic Studies, University of Tasmania, Hobart, TAS 7001, Australia

3 Tasmanian School of Business and Economics, University of Tasmania, Hobart, TAS 7005, Australia

4 Australian National Centre for the Public Awareness of Science, Australian National University, Canberra, Australia

5 School of Marine and Environmental Affairs, University of Washington, Seattle, WA 98105, USA

6 School of Environment, Resources and Sustainability, University of Waterloo, Waterloo, ON N2L3G1, Canada

7 Department Ecosystem Services, Helmholtz-Center for Environmental Research - UFZ, Permoserstraße 15, 04318 Leipzig, Germany

8 Institute of Biodiversity, Friedrich Schiller University, Dornburger Str. 159, 07743 Jena, Germany

9 German Centre for Integrative Biodiversity Research (iDiv), Deutscher Platz 5e, 04103 Leipzig, Germany
10 Fish Ecology and Conservation Physiology Laboratory, Department of Biology, Carleton University, Ottawa, ON, Canada

11 CSIRO Oceans and Atmosphere CSIRO, Hobart Tasmania 7001, Australia

12 National Center for Ecological Analysis and Synthesis, University of California, Santa Barbara, CA 93101, Canada

13 Bren School of Environmental Science and Management, University of California, Santa Barbara 93106, USA

14 Department of Ecology, Universidade Federal do Rio Grande do Norte, Natal, RN, Brazil

15 Department of Zoology, University of Oxford, OX13PS Oxford, UK

16 Institute for Marine Ecosystem and Fishery Science, Center for Earth System Research and Sustainability, University of Hamburg, 22767 Hamburg, Germany

17 Department of Fisheries and Oceans, Biological Station, St. Andrews, NB, Canada

18 Canadian Fisheries Research Network, University of New Brunswick, Saint John, NB, Canada

19 National Marine Fisheries Service, NOAA, Silver Spring, MD 20910, USA 


\section{A need for guidance}

Research and collaboration between the biophysical and human dimensions are fundamental to address modern socio-ecological and sustainability challenges. Disentangling these challenges demands knowledge on complex, multi-scale interactions between ecosystems and society (Nash et al. 2017, pp. 1625-1634) and is an inherently interdisciplinary endeavour that recognises the interdependence of society and the biosphere, from local to global scales (Folke et al. 2016; Coen 2018, p. 41). Interdisciplinary socio-ecological approaches (Fig. 1) are increasingly prevalent within academia (Rhoten and Parker 2004, p. 2046; Campbell 2005, p. 574; Palmer 2018, p. 331) and beyond, particularly illustrated by the conception of the Sustainable Development Goals agenda (United Nations 2015). Despite this, challenges and barriers to conducting interdisciplinary research (IDR) are considerable (Rhoten and Parker 2004, p. 2046; Pooley et al. 2013, pp. 25-28; Hein et al. 2018, pp. 10-11). Research that successfully integrates social, human, health and natural science realms is a rarity (Ommer 2018, p. 1) because the culture, structures and practices necessary to facilitate IDR are lacking.

IDR often requires combining the disparate concepts and methods that diverse disciplines are founded upon (Eigenbrode et al. 2007, p. 56). This requires time and resources and adds to project transaction costs (i.e. defining research approaches, terminology, fundamental disciplinary concepts or ways of thinking, etc.) which can induce destabilising tensions within interdisciplinary teams (Frusher et al. 2014, p. 605). Researchers may be reluctant to engage with IDR because they do not receive recognition for their efforts in their home disciplines (Pain 2014, p. 1) and because academic organisation and culture often penalises IDR (Bromham et al. 2016, pp. 684-687). For example, research institutions are traditionally structured along disciplinary lines and funding proposals that straddle these lines (i.e. falling between natural and social sciences) are largely judged from single disciplinary perspectives, which lack the expertise necessary to assess research proposals outside of their core discipline (Bammer 2016, p. 4).

A further problem is narrowly focused research programmes conducted under the banner of interdisciplinarity (Clark and Steelman 2013, p. 25; Budtz Pedersen 2016, p. 16036). For example, a commonly voiced issue is the late inclusion of a 'token' researcher from a different discipline (often, from the social sciences) after a research programme has been designed, rather than integrating all disciplinary perspectives from inception (Stephenson et al. 2017, p. 58). Increasing awareness of these (among other) impediments to IDR are driving progress towards better support for interdisciplinary teams (Blythe et al. 2017, p. 118; Alexander et al. 2018, p. 72). Still, researchers, particularly those at early career stages, are often uncertain on how best to effectively engage in an interdisciplinary setting (Charnley et al. 2017, p. 81). Thus, this Perspective Essay aims to provide guidance for those engaging, or wishing to engage, in IDR. We draw on the experiential knowledge of world-leading interdisciplinary scholars to articulate 10 tips for early career socio-ecological researchers and their mentors for improving IDR in practice.
Fig. 1 Defining the concepts of multidisciplinarity, interdisciplinarity and transdisciplinarity. Adapted from Stember (1991, p. 5) and Tress et al. (2006, p. 17). This article focuses on interdisciplinarity, as opposed to multidisciplinarity or transdisciplinarity, although there are certainly similarities across the three

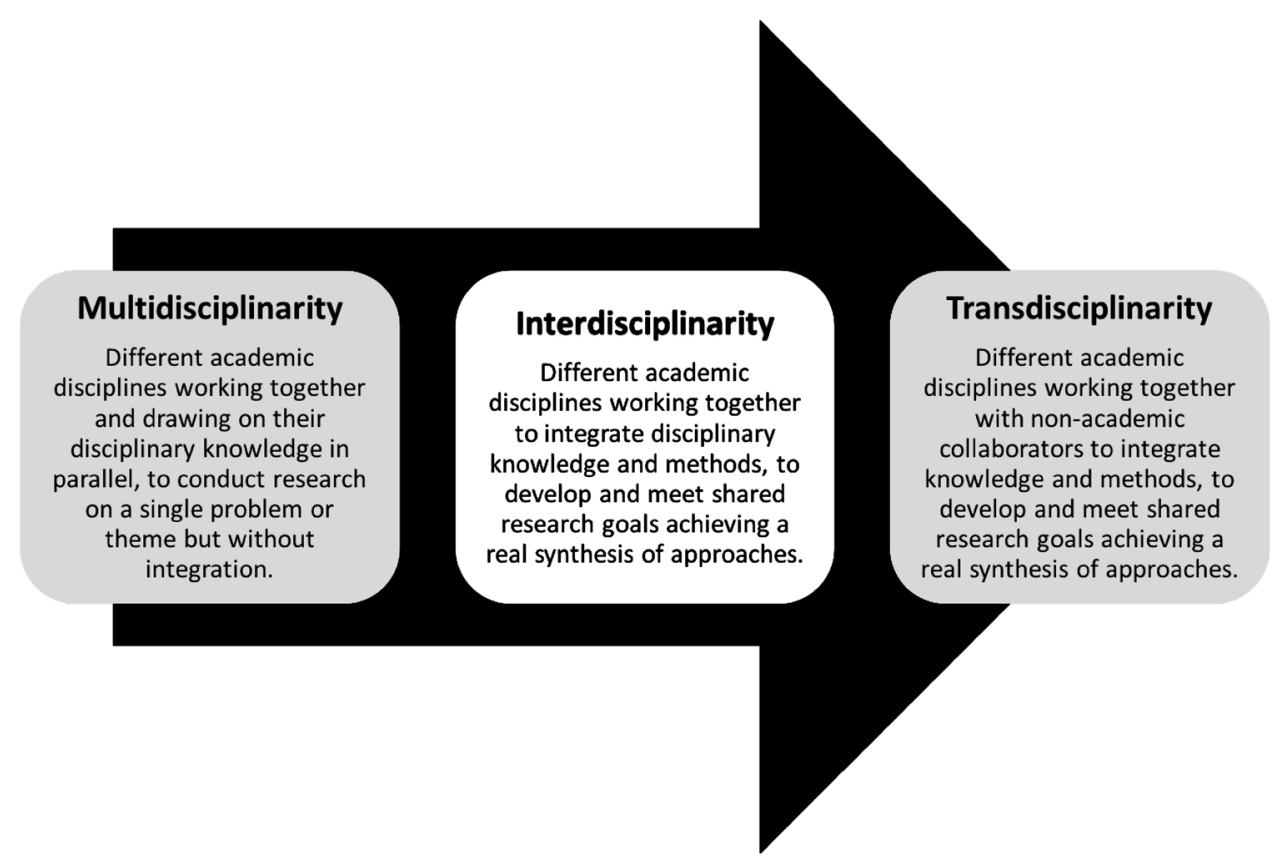




\section{In search of guidance}

\subsection{Identifying interdisciplinary experts}

To address the aims of this paper, the coordinating authors (RK, MM, KLN, CC) identified and engaged with thirteen leading practitioners (other authors) of interdisciplinary approaches from around the globe (hereafter, 'experts'). These experts were selected purposely and opportunistically for five reasons. First, they have diverse and extensive experiences operating and publishing as self-identified IDR researchers. Second, each has extensive experience leading IDR groups and/or teams. Third, they come from a range of disciplinary backgrounds including oceanography, biology, mathematics, geography, sociology and natural resource management. Fourth, they represent several research and practice approaches (e.g. quantitative, qualitative, mixedmethods, participatory approaches). Fifth, they have worked under a range of institutional contexts, including academia, government and non-government agencies, which recognise and combine fundamental worldviews (see e.g. Eigenbrode et al. 2007, p. 56). The experts were identified through the coordinating authors' existing professional networks and, as such, are largely situated in marine and aquatic research domains in English-speaking, developed countries, and are not representative (nor are they intended to be) of the wider population of interdisciplinary experts. However, their diversity of experiences and perspectives should make our interdisciplinary recommendations more generally useful.

\subsection{Collating expertise and advise}

The coordinating authors developed a qualitative questionnaire, designed to encourage the experts to reflect on their careers and to garner insight from their experience that would be beneficial as guidance for (aspiring) ID researchers. Specifically, the first question was designed to understand the experiences of each participant, including how they came to work as an ID researcher. The next three questions were designed to understand their broad experiences working as, and leading teams of, ID researchers including their perceptions as to the need for ID researchers (question 2 ), the challenges and barriers that they had experienced in their careers relating to IDR (question 3), and the strategies that they have used (or seen used) to overcome these challenges (question 4). The next two questions, adapted from Marshall et al. (2017, p. 3), were specifically designed to convert the experiences and learnings of participants into well-constructed advice that could be converted directly into a 'tip' for early career researchers seeking to become interdisciplinary (question 5) and senior researchers seeking to cultivate ID researchers and teams (question 6). The final question (question 7) was included to allow participants to reflect on the survey and provide any additional context or information that they felt was needed, having just completed the survey. The survey questions were:

1. Can you please outline your individual experience? What is your background, and how did you become involved in IDR?

2. In your opinion, why do we need ID researchers?

3. Based on your experiences, what are the main barriers and challenges related to developing ID researchers?

4. In your experience, what strategies have you used, or seen used, to successfully develop ID researchers?

5. If you could give one 'top tip' to students and early career researchers trying to become ID researchers, what would it be?

6. If you could give one 'top tip' to senior researchers and lab leaders responsible for the training and development of ID researchers, what would it be?

7. Given the topic of this paper, is there anything else that you would like to tell us?

The survey was administered between May and July 2018, and each expert had the option of answering the questions via interview $(n=11)$ or providing written responses $(n=2)$. In the case of interviews, we provided the questions to the experts in advance to allow them time to carefully consider their responses and to provide in-depth recollections of their experiences. We conducted the interviews in person where possible or via Skype, and they lasted between 20 and $60 \mathrm{~min}$, were audio recorded and then professionally transcribed.

\subsection{Data analysis}

The transcripts were analysed using NVIVO 10 qualitative data analysis software. The research objectives formed the basis of the coding, and the analysis of the raw data was completed following an inductive approach, based on Grounded Theory Analysis (Glaser and Strauss 1967). This allowed the research findings (key themes) to emerge naturally from the interviews, without the restrictions imposed by more structured methodologies (Hay 2010). To ensure the validity of the emerging themes, they were continually verified against the raw data from which they were derived (following previous studies, e.g. Cvitanovic et al. 2016, p. 886; Marshall et al. 2017, p. 3). Collective author reflection on the themes during the group synthesis and preparation of this paper further verified their relevance and value.

The analysis of the data resulted in the development of 10 tips to help early career researchers, and their mentors achieve successful IDR. We present these 10 tips under a knowledge, attitudes and practices (KAP) framing (Cabana 


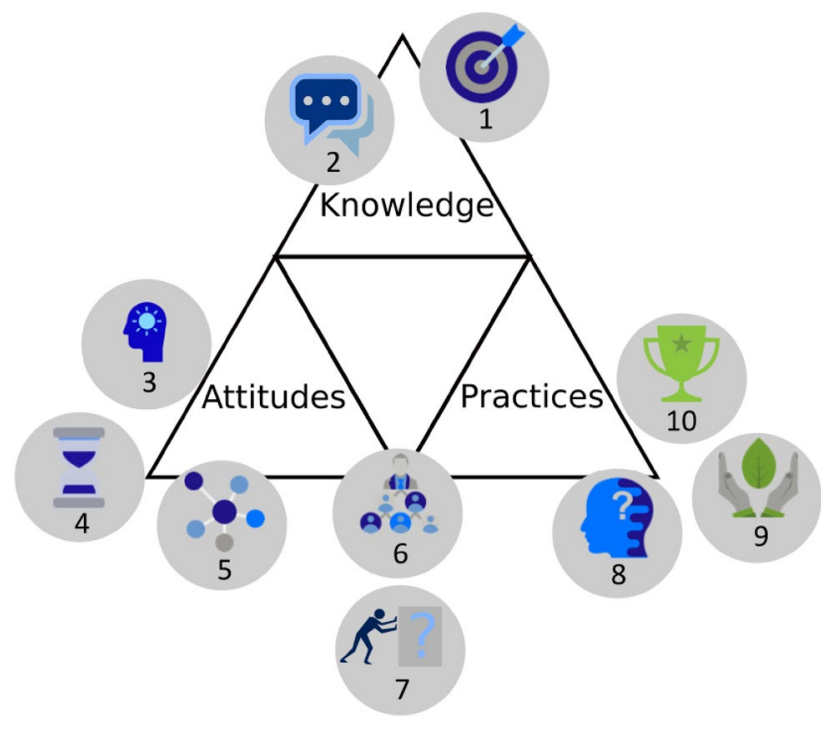

Fig. 2 Ten tips for ID researchers, depicted under the knowledge, attitudes and practices (KAP) framework (Cabana et al. 1999, p. 1459). Tips 1 (Develop an area of expertise) and 2 (Learn new languages) relate to developing ID researcher knowledge. Tips 3 (Be open-minded), 4 (Be patient) and 5 (Embrace complexity) pertain to ID researcher attitudes. Tips 6 (Collaborate widely) and 7 (Push your boundaries) relate to both attitudes and practices. Tips 8 (Consider if (and how) you will engage in IDR), 9 (Foster interdisciplinary culture) and 10 (Champion researchers) emphasise engaging in, and supporting, IDR. Tip 9 (Foster interdisciplinary culture) was the most discussed theme $(n=12)$, followed by tip 6 (Collaborate widely; $n=11$ ) and tip 2 (Learn new languages; $n=11$ ). Tip 10 (Champion interdisciplinary role-models) was the least discussed theme and was highlighted by only one expert. Tips $1-8$ are aimed at early career researchers and are represented by the blue icons, while tips 9-10 are aimed at more senior level researchers, university leaders and mentors and are represented by the green icons.

et al. 1999, p. 1459) to target what researchers should know (knowledge), perceive (attitudes) and do (practices) in relation to conducting IDR (Fig. 2). This adds a further layer of structuring, which we intend to increase the usefulness and applicability of the tips; i.e. behaviour change can be encouraged without changing knowledge and attitudes first; however, impelling behaviour change by influencing knowledge and attitudes is believed to be more successful in the long term (Cabana et al. 1999, p. 1463).

\section{Tips for interdisciplinary researchers}

The 10 tips outlined below are presented in a generic manner to increase their applicability and utility across disciplines, geographies, career stages and contexts. However, as outlined earlier, not all tips will be useful across all contexts and some may be more relevant and useful to students and early career researchers, while others to more senior, mentoring or institutional levels. Specifically, tips 1-8 are aimed at the individual researcher and the final tips, 9-10, are focused more on the interdisciplinary team or research strategy level. The tips are presented in an order that logically accompanies the process of becoming an ID researcher, as opposed to the order of the frequency in which they were discussed (i.e. number of experts). Further, these tips are not mutually exclusive, but rather emphasise the key themes of our analysis, which in many cases reinforce one another. We have chosen not to identify specific expert comments and instead use identity codes (e.g. E1 for expert 1, etc.) in the quotations below.

\subsection{Tips pertaining to knowledge}

\subsection{1} (C) Tip 1: develop an area of expertise-work
on your core

Interdisciplinarity means bridging between disciplines, but a core grounding is required to bring an expert perspective to the interdisciplinary table comfortably, confidently and most importantly, competently.

"As oddly contrasting as the terms might be, I think to be interdisciplinary, you also have to be a specialist at something and find that balance." (E3)

This 'core' knowledge can be either a discipline (e.g. marine zoology), a place (e.g. having a deep experiential understanding of the Arctic), a field of study (e.g. fisheries), a method (e.g. modelling) or a process (e.g. knowledge brokering). A core knowledge provides a clear identity and profile. It will shape the researcher's contribution and be a stronghold in interdisciplinary collaborations because developing in-depth knowledge promotes an appreciation of the expertise of others.

Note: Several of the experts believe that 'core' knowledge is contextual and that core grounding can also be IDR. Training as a 'core' ID researcher from the beginning of a research career is challenging, but opportunities for early career researchers to engage in interdisciplinary training are increasing and improving, as we discuss in the next section.

\subsection{2 $\because$ Tip 2: learn new languages-seek 2 to understand and speak across disciplines}

IDR requires expression of disciplinary science in ways that are understandable to other disciplines. We are trained to use jargon because it is specific and exact within our fields and/ or disciplines, but in IDR, this jargon will be confusing and excluding. Differences in the use of terms and techniques 
by various disciplines (e.g. specific statistics, mathematical approaches, qualitative methods, etc.) can also generate confusion and misunderstanding, and the use of discipline-specific terms (e.g. 'significance', 'culture', 'function', 'model', etc.) has great potential to marginalise potential collaborators.

In developing a shared language, the challenge arises in not losing the rigour and nuance of terms, as used in a core discipline. Casual and clumsy use of language can promote, and be a symptom of, clumsy thinking. Just as learning a different (e.g. national) language requires an understanding of grammar, nuance and meaning, learning a different disciplinary language also requires this grammatical and cultural understanding. Superficial ability to function and communicate may come relatively quickly, but fluency takes time, patience and immersion. In the long term, working through language barriers will increase flexibility and adaptability to work across disciplines and can foster the creation of IDR questions and solutions.

"We need the people who can span the disciplines and kind of speak both languages, sit in that boundary as service translators or be able to think within different frames of reference and thinking as it's really valuable and it is a unique skill set (E5)".

Learning new languages can best be achieved by listening, questioning and more listening. Sharing disciplinary definitions or key introductory texts may be helpful. Communication tools, such as metaphors, analogies and stories, may also be used to present experiences and perspectives to diverse audiences and disciplines. Communication experts and knowledge brokers can facilitate dialogue between disciplines to break down language barriers and support understanding and collaboration.

\subsection{Tips pertaining to attitudes}

3.2.1

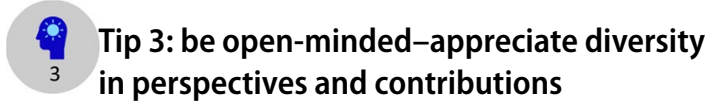

IDR necessitates integrating divergent disciplines, and navigating this can be intimidating and even lead to confrontation, particularly for researchers who have been taught within the norms and rules of a single discipline. The key to becoming a successful ID researcher is to remain openminded; open to learning, open to new ways of doing things and open to collaborations that include new types of disciplinary knowledge and non-academic knowledge. Most importantly, one is to remain humble when engaging with other knowledge-holders. Invite questions, ask them to explain and never be afraid to say 'I don't understand'.
"People who are humble probably do [IDR] better and more rapidly than people who aren't humble". (E2)

\subsection{2}

\section{Tip 4: be patient-IDR takes time}

Some collaborations work more easily than others, but most frequently, establishing successful interdisciplinary collaborations requires time, and lots of it. IDR is a learning cycle, and transaction time can lengthen when collaborators need to understand different disciplinary cultures, languages and approaches. Time should be allocated for iterative cycles of learning and reflection across all stages of the research process: from the development of the research questions to the solutions that are proposed:

"Being patient and allowing everybody to learn. I think having patience to allow new kinds of working and being open really, and curious". (E1)

Successful IDR is underpinned by trust among members of the research team, and trust-building takes time because IDR can be an uncomfortable, frustrating space requiring lengthy social bonding processes and effort and patience from researchers. This need for time is one of the main reasons early career stage researchers may find it challenging to engage in IDR, because the academic reward system emphasises the regular and rapid production of publications, particularly in the early stages of a researcher's career.

\subsection{3}

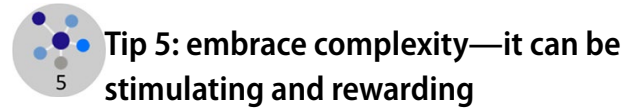

Do not view complexity and differences in approaches as a roadblock. Rather, embrace this complexity and appreciate that every researcher will make a contribution. It would be naïve to underestimate the true complexity of the disciplinary cores you and others are representing, or the complexity of the socio-ecological challenges being tackled. IDR will be difficult, but consider its complexity and associated ambiguity as opportunities rather than barriers. For example, working to understand the 'bigger picture', by combining views and knowledge from several disciplines, provides a richer perspective:

“There's been a bunch of times where I'm reading a paper from another discipline or struggling through that terminology and suddenly go, 'Oh wow, I never would have thought of that'. You find common patterns across scales that you wouldn't have appreciated otherwise, you can open up doorways". (E2) 
Research questions should include input and insight from all members of the research team to provide a clear and shared focus for all, facilitating the contribution of all participants' critical role.

\subsection{Tips pertaining to attitudes and practices}

3.3.1

\section{Tip 6: collaborate widely—but check your ego} in at the door

Interdisciplinary work is integrated and collaborative: bringing people together to harness and discuss their collective expertise. Egos will impede progress because IDR requires hearing and appreciating the views and knowledge of others. Learning nuances and differences in thought and approaches among disciplines is crucial for co-creating research questions and approaches to answer them. Building teams of engaged collaborators spanning multiple disciplines is a powerful strategy for advancing understanding of important socio-ecological challenges. Collaborating broadly fosters joint learning, a significant reward for undertaking IDR, and interdisciplinary team members share collective interest in working on the challenge at hand and in learning from other researchers:

“Take the journey together...it's not a relay race. It's not like, 'I did something and now I'm going to give you the baton, and now you run the next hundred metres to hand the baton to someone else'. It really should not be a relay race". (E3)

\subsection{2 $\digamma^{2} \quad$ Tip 7: push your boundaries-get comfortable 7 outside your comfort zone}

IDR provides opportunity to question how you understand things and to challenge yourself to comprehend something differently. Make attempts, big and small, to get outside of your comfort zone, and deliberately expose yourself to novel perspectives, opinions and ideas:

"There are whole bodies of theory there that I' $m$ completely ignorant of, and I know I'm ignorant of them. I think in some ways you've got to be quite brave and bold to be interacting in these spaces where you know you know nothing". (E7)

For example, read and attend seminars outside of your discipline, and discuss and share what you learn among your peers. Broadening your disciplinary perspective through IDR can prove enlightening and promote novel and innovative approaches to tackling complex research challenges.

\subsection{Tips pertaining to practices}

3.4.1

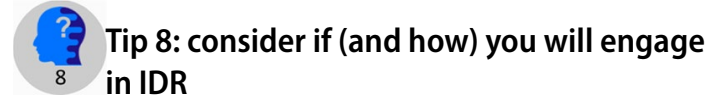

Interdisciplinary career paths will not appeal to everyone nor do they need to. Many researchers address critical questions within their disciplinary boundaries. Personal skill sets and outlooks will differ between those who aspire to conduct IDR and those who prefer to remain within a single discipline.

'Some people want to keep pursuing their [single discipline] and they're very, very good at it, and that's the best road for them'. (E1)

It is important to identify personal aspirations and skills and to consider whether IDR aligns with these career and personal goals. IDR is challenging and requires patience and perseverance, but can provide complex and compelling solutions to the difficult questions and 'wicked' problems around us.

Tip 9: foster interdisciplinary culture-support researchers at the grassroots level

Institutional leaders and senior researchers should foster open atmospheres and safe spaces where interdisciplinary work can be discussed and developed, i.e. where saying 'I don't understand' is supported. Lab leaders should be ready to challenge researchers to explain their jargon and engage and invite researchers from across disciplines and perspectives into their lab group meetings and events. Achieving interdisciplinary culture relies on lab groups and researchers having the freedom to think and work across disciplinary boundaries.

Institutional leaders can work to adapt internal cultures within their organisations, to ensure that interdisciplinary work is valued, resource allocation (e.g. of time, meeting space and finances) can be granted, and that the formal internal and external recognition necessary for career progression can be provided. Informal encouragement could involve assisting early career researchers to take opportunities that support interdisciplinary skill development or discussing the advantages (and pitfalls) of IDR openly and frequently.

"Connect them to as many good collaborative people in the different disciplines as possible, so that they've got that supportive network from the start". (E2)

More formal support could involve developing adequate training programmes, and ensuring that these opportunities are more accessible to students across all disciplines who want to understand and practise IDR. 


\section{4 .3 Tip 10: champion researchers-showcase 10 examples of interdisciplinary success}

Great IDR deserves recognition akin to that awarded within disciplines for academic contribution and practical output. Leaders and champions can create opportunities and remove barriers by creating and promoting high-profile awards and developing other mechanisms to recognise excellence in IDR. Praise and recognition are central to improving researchers' track records, while they incur the transaction costs associated with working across disciplines. Rewards may also motivate otherwise reluctant disciplinary researchers to participate in interdisciplinary work.

"I've seen people succeed in interdisciplinarity with very, very different strategies. I think there isn't one way, I think there are multiple pathways". (E5)

\section{Use of the 10 tips in practice}

Training disciplinary experts to collaborate together in interdisciplinary teams is necessary and useful, but in light of the complex socio-ecological challenges society faces, we must also focus on developing ID researchers (Alexander et al. 2018, p. 77). In the previous section, we identified particular knowledge, skills, practices, attitudes and/or personality traits which researchers need if they hope to effectively work across disciplines. These include humility, respectfulness, open-mindedness, patience and a disposition to work with others in challenging situations. Indeed, while such 'soft skills' are consistently identified as critical for collaborations among disciplines and knowledge systems (e.g. Evans and Cvitanovic 2018, p. 8), they are rarely valued or specifically recognised and developed within most existing postgraduate research training.

Effective and successful IDR requires careful reflection and dedicated effort by the consortium of researchers involved and their institutions (Lyall and Meagher 2012, p. 616; Schwartz et al. 2017, p. 587). We highlight the KAP framework as a means to achieving this. By framing our '10 Tips' as components of what researchers should know (knowledge), perceive (attitudes) and do (practices) in conducting IDR, we have identified a context within which an (aspiring) ID researcher can employ each tip. For instance, if researchers are supported (Tip 9) in learning new terms, techniques and approaches (Tip 2), and being open-minded (Tip 3), the diversity of disciplinary 'languages' may evolve from a barrier to a starting point for fruitful and exciting research. Knowledge sharing can lead to the creation of new ideas which can advise new understanding (i.e. attitudes), and these will inform the development of IDR practices and designs, which can include new methods and institutions.

Encouraging reflection and effort are unlikely without paying explicit focus to the development of ID researchers and to the cultivation of supportive labs and research groups, and broader institutional culture (Palmer 2018, p. 331). Challenging deeply held disciplinary beliefs provides opportunities for dialogue, growth and innovation (Campbell 2005, p. 575; Schwartz et al. 2017, p. 591). Such focus fosters the potential to address many of the challenges of conducting IDR in practice, even in instances where structural and institutional barriers remain (Eigenbrode et al. 2007, pp. 61-62) (Table 1).

As the research climate evolves to provide more opportunities to conduct IDR and afford better recognition of its outcomes, Ph.D. students and early career researchers will benefit from purposeful engagement with interdisciplinary training and communication, that can broaden perspectives beyond highly specialised home disciplines (Bridle et al. 2013 , p. 27). Increasingly, there is a view that interdisciplinary training should begin in the early career stages (Roy et al. 2013, p. 750). Today's graduate students increasingly commence their careers with an interdisciplinary background (Haider et al. 2018, p. 192), and we posit that the 10 tips can inform the design of interdisciplinary graduate courses. Developing an interdisciplinary 'core' (Tip 1) will provide early career researchers with the skills necessary to develop and facilitate collaborations when needs and challenges arise, as well as the expertise to combine and integrate diverse knowledge for purposeful impact.

Interdisciplinary courses have the potential to provide a robust graduate foundation that can produce effective modern researchers and agents of change. Opportunities for formal university training opportunities are increasing (Rhoten and Parker 2004, p. 2046; Thiet et al. 2018, p. 314), however, remain rare because academic staff with adequate interdisciplinary training and backgrounds are a rarity in themselves (Turgeon et al. 2017, p. 8). Interdisciplinary programmes are structured in myriad ways but tend to be problem- and solution-focused, not discipline-based (Klein 2005, pp. 35-44; Thompson et al. 2019, pp. 671-681), with a range of expectations as to what should constitute 'interdisciplinary training' (Meyer et al. 2016, p. 348). Perhaps early career stage researchers are best placed to revolutionise traditional approaches to research (Chapman et al. 2015, p. 342). Giving adequate attention to the processes of collaboration and communication involved in interdisciplinary experiences (Tips 2 and 6) will be a critical component of any course developing interdisciplinary skills (Bridle et al. 2013, p. 30).

IDR combines previously disconnected ideas, concepts and resources, and often violates disciplinary expectations to produce novel ideas with high impact (Fortunato et al. 
Table 1 Use of the 10 tips in practice. We identify commonly cited barriers to IDR from the literature and identify which tips can be used to overcome them, in addition to providing some examples of implementing the tips in practice

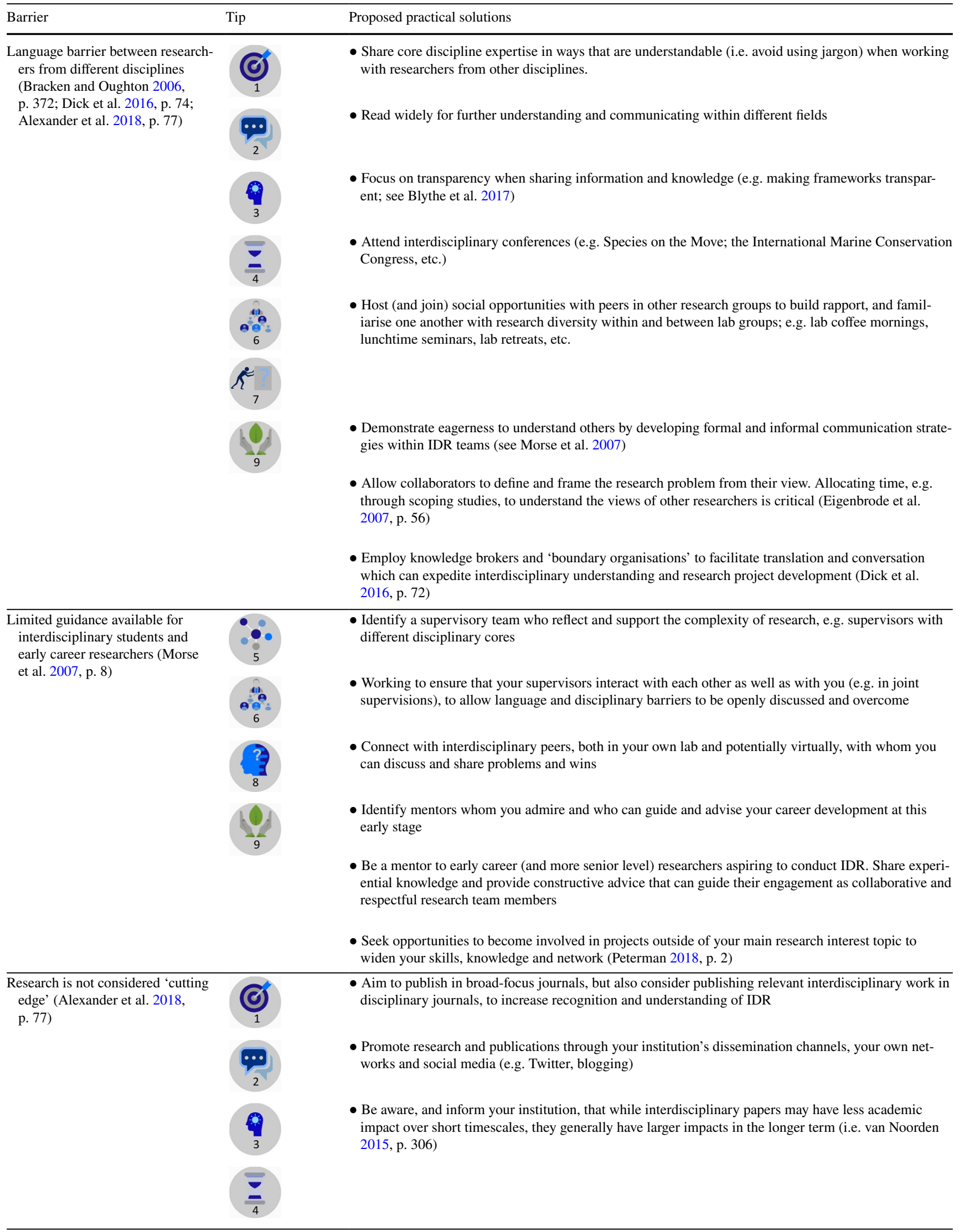


Table 1 (continued)

\begin{tabular}{|c|c|c|}
\hline Barrier & Tip & Proposed practical solutions \\
\hline & & $\begin{array}{l}\text { - Recognition breeds recognition. Support and highlight successful ID researchers in your group, and in } \\
\text { other groups, for their 'cutting edge' research to increase awareness and appreciation } \\
\text { - If you admire IDR published by others, share this with your networks and on your public channels; } \\
\text { mutual respect is contagious }\end{array}$ \\
\hline $\begin{array}{l}\text { Significant transaction costs (Brom- } \\
\text { ham et al. 2016, pp. 684-687) }\end{array}$ & & $\begin{array}{l}\text { - Collaborate widely but draw a line that maintains a good work-life balance. } \\
\text { - Seek projects that can both challenge and reward you personally (e.g. international collaborations, } \\
\text { projects with potential to continue into the future, etc.) } \\
\text { - Resolve discipline-specific conceptual differences while developing project ideas, to best utilise lim- } \\
\text { ited time and resources (Pooley et al. 2013, p. 29) }\end{array}$ \\
\hline $\begin{array}{l}\text { Publishing IDR can be difficult } \\
\text { (Dick et al. 2016, p. 74) }\end{array}$ & & $\begin{array}{l}\text { - Collaborating across networks and disciplines can enhance research visibility via a larger network of } \\
\text { co-authors (Fortunato et al. 2018, p. 3), increasing academic (and other) impact } \\
\text { - Be patient. Interdisciplinary teams tend to achieve higher rates of publication than single discipline } \\
\text { teams in the long term (Hall et al. 2012, p. 160) } \\
\text { - Provide disciplinary 'anchors' and definitions in your publications, so that IDR can also be understood } \\
\text { (and referenced) by single disciplinary colleagues }\end{array}$ \\
\hline $\begin{array}{l}\text { Fewer funding opportunities } \\
\text { (Campbell 2005, p. 575; Roy } \\
\text { et al. 2013, pp. 745-746; Brom- } \\
\text { ham et al. 2016, pp. 684-687) }\end{array}$ & & $\begin{array}{l}\text { Funding opportunities are increasing, but in the interim: } \\
\text { - Apply to broad-focus and/or philanthropic funding opportunities (i.e. Belmont Forum, Horizon 2020, } \\
\text { G8 Research Councils Initiative, etc.) } \\
\text { - Pool research funding within teams; i.e. from different disciplinary funding schemes } \\
\text { - Share and promote information about IDR funding opportunities with your networks (e.g. US } \\
\text { National Science Foundation, Australian Research Council, EU Research Council, etc.) }\end{array}$ \\
\hline $\begin{array}{l}\text { Lack of credit given to IDR may } \\
\text { hinder career development (Rho- } \\
\text { ten and Parker 2004, p. 2046; } \\
\text { Roy et al. 2013, p. 749) }\end{array}$ & & $\begin{array}{l}\text { - Work to champion researchers who perform interdisciplinary work can strengthen the track record and } \\
\text { legitimacy of IDR } \\
\text { - Pursue interdisciplinary networking opportunities (i.e. conferences and seminars, or Twitter) to facili- } \\
\text { tate collaboration (Chapman et al. 2015, p. 343) } \\
\text { - Present at conferences both which are on the margins of your experience and comfort zone, as well as } \\
\text { conferences in your 'core field' to promote novel ideas and spark new collaborations }\end{array}$ \\
\hline $\begin{array}{l}\text { Researchers viewed as 'Jack of all } \\
\text { trades' (Morse et al. 2007, pp. } \\
\text { 18-19) }\end{array}$ & & $\begin{array}{l}\text { - Remain confident and assured in the value and relevance of IDR. Demonstrated expertise in a core } \\
\text { area will facilitate this } \\
\text { - Reading broadly across disciplines, collaborating widely in research and choosing projects that are } \\
\text { challenging and rewarding will help. The tide is turning! }\end{array}$ \\
\hline
\end{tabular}


2018, p. 2). IDR experiences can be further rewarding when ID researchers share collective interest in learning and sharing and understanding new perspectives. However, it is also very challenging, particularly where disciplines do not share fundamental concepts or approaches (Eigenbrode et al. 2007, p 57; Morse et al. 2007, p. 18). An interdisciplinary way is not the only way; not all researchers want (or need) to be interdisciplinary, and single disciplinary research themes and approaches are crucially important (Schwartz et al. 2017, p. 595). Conducting research to attain in-depth knowledge and understanding of the world around us is among the many reasons why individual disciplines exist (Ommer 2018, p. 3 ), and successful, credible IDR requires solid disciplinary knowledge (Wu 2006, p. 4). A balance of disciplinary expertise and interdisciplinary integration is necessary for effective socio-ecological research in practice (Roy et al. 2013, p. 751).

\section{Conclusions}

Socio-ecological research is not easily contained within one field or domain nor are research questions adequately formulated, addressed or resolved using single disciplinary approaches. Single discipline research and teaching still predominate in academia (Lyall and Meagher 2012, p. 608) and will continue to play an important role in the pursuit of knowledge. However, the research landscape is changing. Our 10 tips, which draw on the extensive expertise of some IDR leaders, convey tangible and readily implementable actions. They have been compiled to support early career and more senior level researchers, their mentors and institutions who wish to embrace interdisciplinarity. We hope that these 10 tips can improve researcher capacity to communicate across a range of disciplines and catalyse insights that successfully integrate knowledge, to produce exciting and novel strategies for addressing global socio-ecological and sustainability challenges.

\section{References}

Alexander KA, Hobday AJ, Cvitanovic C, Ogier E, Nash K, Cottrell RS, Fleming A, Fudge M, Fulton EA, Frusher S, Kelly R, Macleod C, Pecl GT, van Putten EI, Vince J, Watson RA (2018) Progress in integrating natural and social science in marine ecosystem-based management research. Mar Freshw Res 70:71-83

Bammer G (2016) What constitutes appropriate peer review for interdisciplinary research? Palgrave Commun 2:16017

Blythe J, Nash K, Yates J, Cumming GS (2017) Feedbacks as a bridging concept for advancing transdisciplinary sustainability research. Curr Opin Environ Sustain 26-27:114-119

Bracken LJ, Oughton EA (2006) 'What do you mean?' The importance of languge in developing interdisciplinary research. Trans Inst $\mathrm{Br}$ Geogr 31:371-382
Bridle H, Vrieling A, Cardillo M, Araya Y, Hinojosa L (2013) Preparing for an interdisciplinary future: a perspective from early-career researchers. Futures 53:22-32

Bromham L, Dinnage R, Hua X (2016) Interdisciplinary research has consistently lower funding success. Nature 534:684-687

Budtz Pedersen D (2016) Integrating social sciences and humanities in interdisciplinary research. Palgrave Commun 2:16036

Cabana MD, Rand CS, Powe NR, Wu AW, Wilson MH, Abboud PAC, Rubin HR (1999) Why don't physicians follow clinical practice guidelines? A framework for improvement. JAMA 282:1458-1465

Campbell LM (2005) Overcoming obstacles to interdisicplinary research. Conserv Biol 19:574-577

Chapman JM, Algera D, Dick M, Hawkins EE, Lawrence MJ, Lennox RJ, Rous AM, Souilere CM, Stemberger HLJ, Struthers DP, Vu M, Ward TD, Zolderdo AJ, Cooke SJ (2015) Being relevant: practical guidance to early career researchers interested in solving conservation problems. Glob Ecol Conserv 4:334-348

Charnley S, Carothers C, Satterfield T, Levine A, Poe MR, Norman K, Donatuto J, Breslow SJ, Mascia MB, Levin PS, Basurto X, Hicks CC, García-Quijano C, St. Martin K (2017) Evaluating the best available social science for natural resource management decisionmaking. Environ Sci Policy 73:80-88

Clark SG, Steelman TA (2013) Interviewing for an interdisiciplinary job: principled goals, pragmatic outcomes, and finding the right fit in acadmia. J Environ Stud Sci 3:21-29

Coen DR (2018) Climate in motion: science, empire and the problem of scale. The University of Chicago Press, Chicago

Cvitanovic C, McDonald J, Hobday AJ (2016) From science to action: principles for undertaking environmental research that enables knowledge exchange and evidence-based decision-making. J Environ Manag 183:864-874

Dick M, Rous AM, Nguyen VM, Cooke SJ (2016) Necessary but challenging: multiple disciplinary approaches to solving conservation problems. FACETS 1:62-82

Eigenbrode SD, O'Rourke M, Wulfhorst JD, Althoff DM, Goldberg CS, Merrill K, Morse W, Nielsen-Pincus M, Stephens J, Winowiecki L, Bosque-Pérez NA (2007) Employing philsophical dialogue in collaborative science. Bioscience 57:55-64

Evans MC, Cvitanovic C (2018) An introduction to achieving policy impact for early career researchers. Palgrave Commun 4:88. https://doi.org/10.1057/s41599-018-0144-2

Folke C, Biggs R, Norström AV, Reyers B, Rockstrom J (2016) Socioecological resilience and biosphere-based sustainability science. Ecol Soc 21(3):41. https://doi.org/10.5751/ES-08748-210341

Fortunato S, Bergstrom CT, Börner K, Evans JA, Helbing D, Milojevič S, Petersen AM, Radicchi F, Sinatra R, Uzzi B, Vespignani A, Waltman L, Wang D, Barabási A-L (2018) Science of science. Science. https://doi.org/10.1126/science.aao0185

Frusher S, Hobday AJ, Jennings S, Creighton C, D'Silva D, Haward M, Holbrook NJ, Nursey-Bray M, Pecl GT, van Putten EI (2014) The short history of research in a marine climate change hotspot: from anecdote to adaptation in south-east Australia. Rev Fish Biol Fisheries 24:596-611

Glaser B, Strauss A (1967) The discovery of grounded theory: strategies for qualitative research. Aldine, Chicago

Haider LJ, Hentati-Sundberg J, Giusti M, Goodness J, Hamann M, Masterson VA, Meacham M, Merrie A, Ospina D, Schill C, Sinare H (2018) The undisciplinary journey: early-career perspectives in sustainability science. Sustain Sci 13:191-204

Hall KL, Stokols D, Stipelman BA, Vogel AL, Feng A, Masimore B, Morgan G, Moser RP, Marcus SE, Berrigan D (2012) Assessing the value of team science: a study comparing center- and investigaro- initiated grants. Am J Prev Med 42:157-163 
Hay I (2010) Qualitative research methods in human geography. Oxford University Press, North York

Hein CJ, Ten Hoeve JE, Gopalakrishnan S, Livneh B, Adams HD, Marino E, Weiler CS (2018) Overcoming early career barriers to interdisciplinary climate change research. WIREs Clim Change 9:e530

Klein JT (2005) The discourse on transdisciplinarity: an expanding global field. In: Klein KT, Grossbacker-Mansuy W, Haberli R, Bill A, Scholz RW, Welti W (eds) Transdisciplinarity: joint problem solving among science, technology and society: an effective way for managing complexity. Birkhauser, Basel, Switzerland, pp 35-44

Lyall C, Meagher LR (2012) A masterclass in interdisciplinarity: research into practice in training the next generation of interdisciplinary researchers. Futures 44:608-617

Marshall N, Adger N, Attwood S, Brown K, Crissman C, Cvitanovic C, De Young C, Gooch M, James C, Jessen S, Johnson D, Marshall P, Park S, Wachenfeld D, Wrigley D (2017) Empirically derived guidance for social scientists to influence environmental policy. PLoS ONE 12:e171950

Meyer SR, Levesque VR, Hutchins Bieluch K, Johnson ML, McGreavy B, Dreyer S, Smith H (2016) Sustainability science graduate students as boundary spanners. J Environ Stud Sci 6:344-353

Morse W, Nielsen-Pincus M, Force J, Wulfhorst JD (2007) Bridges and barriers to developing and conducting interdisicplinary graduatestudent team research. Ecol Soc 12:1-14

Nash KL, Cvitanovic C, Fulton EA, Halpern BS, Milner-Gulland EJ, Watson RA, Blanchard J (2017) Planetary boundaries for a blue planet. Nat Ecol Evol 1:1625-1634

Ommer RE (2018) Curiosity, interdisciplinarity, and giving back. ICES J Mar Sci 75:1526-1535

Pain E (2014) Better recognition for multidisciplinary research. Sci Mag. https://doi.org/10.1126/science.caredit.a1400183

Palmer L (2018) Meeting the leadership challenges for interdisciplinary environmental research. Nat Sustain 1:330-333

Peterman RM (2018) Continuous learning, teamwork and lessons for young scientists. ICES J Mar Sci 76:28-40

Pooley SP, Mendelsohn JA, Milner-Gulland EJ (2013) Hunting down the chimera of multiple disciplinarity in conservation science. Conserv Biol 28:22-32

Rhoten D, Parker A (2004) Risks and rewards of an interdisciplinary research path. Science 306:2046

Roy ED, Morzillo AT, Seijo F, Reddy SMW, Rhemtulla JM, Milder J, Kuemmerle CT, Martin SL (2013) The elusive pursuit of interdisicplinarity at the human-environment interface. Bioscience 63:745-753

Schwartz M, Hiers K, Davis FW, Garfin GM, Jackson ST, Teranso AJ, Woodhouse CA, Morelli TL, Williamson MA, Brunson MW (2017) Developing a translational ecology workforce. Front Ecol Environ 15:587-596

Stember M (1991) Advancing the social sciences through the interdisciplinary enterprise. Soc Sci J 28:03623319

Stephenson RL, Benson AJ, Brooks K, Degnbol P, Dichmont CM, Kraan M, Pascoe S, Paul SD, Rindorf A, Wiber M (2017) Practical steps toward integrating economic, social and institutional elements in fisheries policy and management. ICES J Mar Sci 74:1981-1989

Thiet R, Dawson A, Fagan B, Hubbard J, Lamonde S, Meier T, Settele R (2018) Advice to master's students for successfully navigating an interdisciplinary environmental studies program. Bull Ecol Soc Am 99:314-326

Thompson SA, Stephenson RL, Rose GA, Paul SD (2019) Collaborative fisheries research: the Canadian Fisheries Research Network experience. Can J Fish Aquat Sci 76:671-681

Tress B, Tres G, Fry G (2006) From landscape research to landscape planning: aspects of integration, education and application. Springer, Berlin

Turgeon K, Hawkshaw SCF, Dinning KM, Quinn BK, Edwards DN, Wor C, Parlee CE, Debertin A, Hawkshaw M, Nelson BW, Zhang F, Benestan L, Angel E, Morse BL, Mombourquette D (2017) Enhancing fisheries education and research through the Canadian
Fisheries Research Network: a student perspecitve on interdisciplinarity, collaboration and inclusivity. FACETS 3:1-17

UN General Assembly (2015) Transforming our world: the 2030 agenda for sustainable development, 21 October 2015, A/RES/70/1. Available at: https://www.refworld.org/docid/57b6e3e44.html

van Noorden R (2015) Interdisciplinary research by the numbers. Nature 525:306-307

Wu JJ (2006) Landscape ecology, cross-disciplinarity, and sustainability science. Landsc Ecol 21:1-4

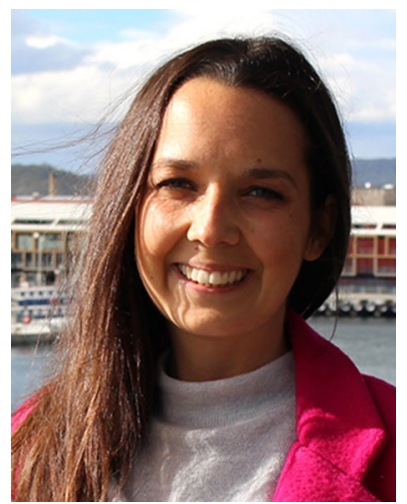

Rachel Kelly is a Ph.D. student at the Centre for Marine Socioecology and Institute for Marine and Antarctic Studies in Tasmania working to improve public engagement with the ocean and marine science. Her research focuses on the human dimensions of marine conservation, including social licence, ocean literacy, marine citizenship and citizen science.

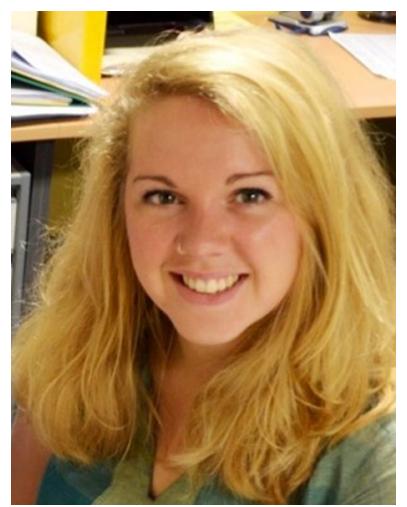

Mary Mackay is a Ph.D. student at the Centre for Marine Socioecology and Tasmanian School of Business and Economics. Her work uses findings from behavioural economics and applies it to incentivising compliance behaviour in fisheries. Her research focuses on testing tools to encourage voluntary compliance and reduce illegal fishing.

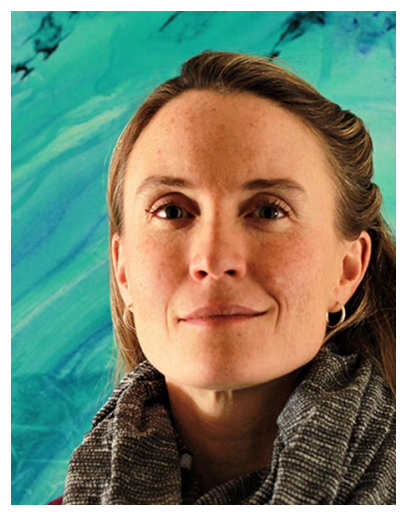

Kirsty L. Nash is a Research Fellow at the Centre for Marine Socioecology and the Institute for Marine and Antarctic Studies at the University of Tasmania. Her research focuses on the resilience of marine socio-ecological systems, functional ecology and facilitating interdisciplinary research. 


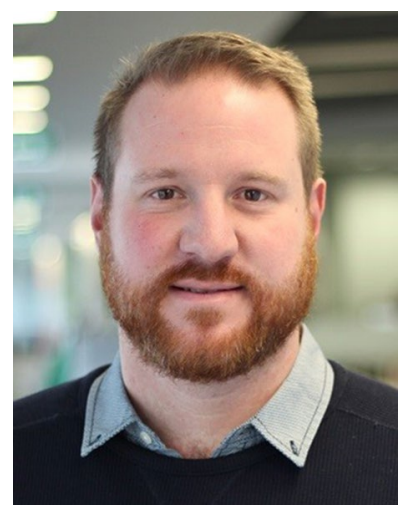

Dr. Christopher Cvitanovic is an environmental social scientist from the Australian National Centre for the Public Awareness of Science at the Australian National University, working to improve the relationship between science, policy and practice to enable evidence-informed decision-making for sustainable ocean futures.

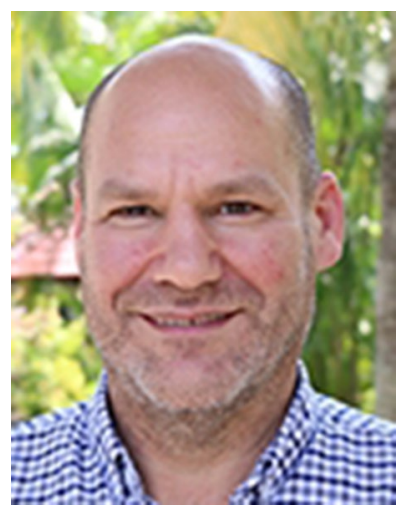

Edward H. Allison is a Professor in the School of Marine and Environmental Affairs at the University of Washington Seattle, WA, USA. His research centres on the human connection to natural resources. His primary focuses are: contribution of fisheries and aquaculture to food and nutrition security and coastal livelihoods; governance of small-scale fisheries and aquaculture production and the human rights of fishers; and vulnerability and adaptation to climate change.

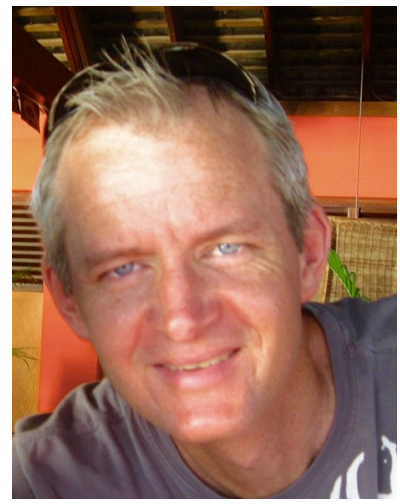

Derek Armitage is Professor in the School of Environment, Resources and Sustainability at the University of Waterloo, Canada. His research focuses on the human dimensions of environmental change, conservation and development and the governance of socio-ecological systems.

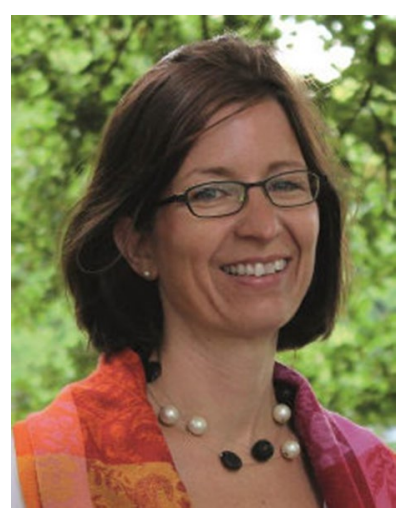

Prof. Aletta Bonn is interested in ecosystem services and biodiversity research and works in the fields of participatory conservation, global change and knowledge management. She relishes challenges at the science-policy interface and values opportunities for joint-working with experts, from society and science, to achieve societally relevant biodiversity and ecosystem service research.

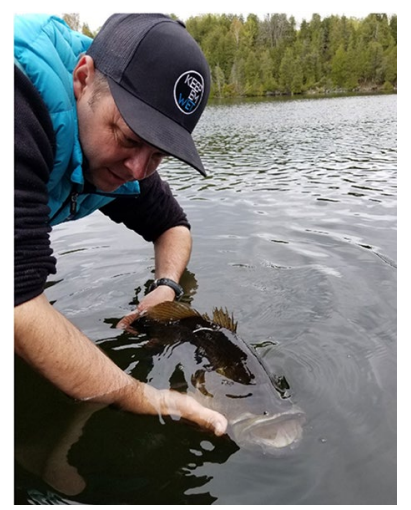

Steven J. Cooke is a Canada Research Chair with deep interests in applied ecology related to aquatic ecosystem. He works closely with diverse partners to conduct research needed to solve complex environmental problems. Cooke draws upon training in the natural and social sciences to ensure that knowledge generated by his team is relevant to end-users.

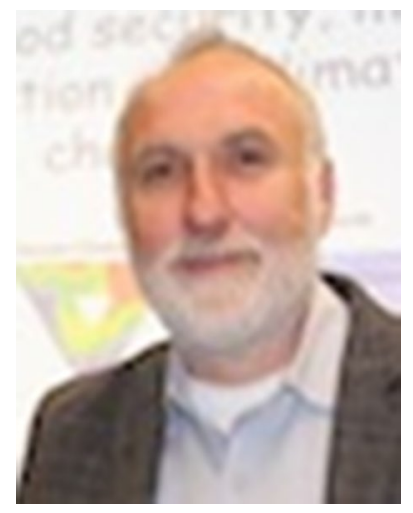

Adjunct Professor Stewart Frusher has developed and led strong interdisciplinary teams and was the inaugural director of the Centre for Marine Socioecology. Recent outcomes include co-development of Australia's Blue Economy CRC that is embedded in a trans-disciplinary and trans-sector approach to enhance seafood and marine renewable energy production.

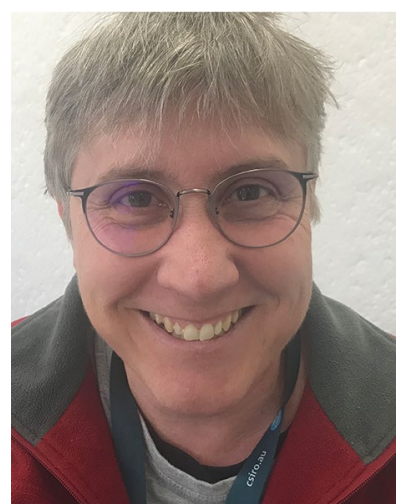

Dr. Elizabeth A. Fulton is a Principal Research Scientist with CSIRO Oceans and Atmosphere where she leads the Marine Ecosystem Modelling and Risk Assessment Group. Beth is also an Adjunct Professor at the Centre of Marine Socioecology. Beth's work focuses on sustainably managing marine environments and adaptation to global change.

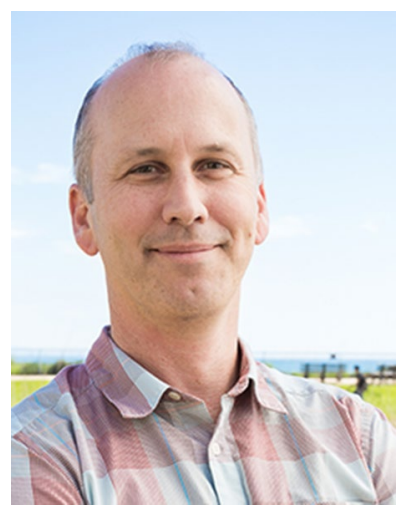

Dr. Benjamin S. Halpern is the Director of the National Center for Ecological Analysis and Synthesis (NCEAS) and Professor in the Bren School of Environmental Science and Management at UC Santa Barbara. His transdisciplinary research addresses the status of global oceans and how to strategically manage and protect them. 


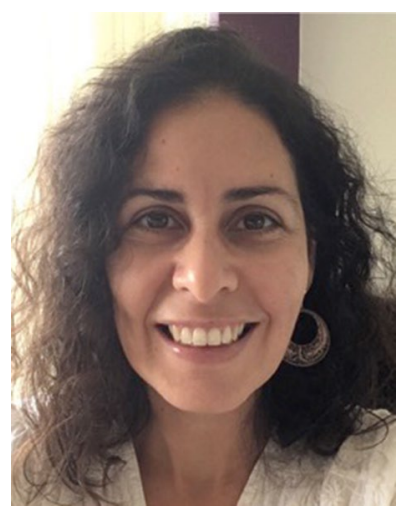

Dr. Priscila F. M. Lopes is an Associate Professor at the Federal University of Rio Grande do Norte, Brazil. She received her B.A. in Biology from the University of Campinas (Brazil) in 2002 and earned her M.Sc. and $\mathrm{Ph} . \mathrm{D}$. in Ecology from the same University. Her research centres around the benefits to society and ecological impacts of smallscale fisheries.

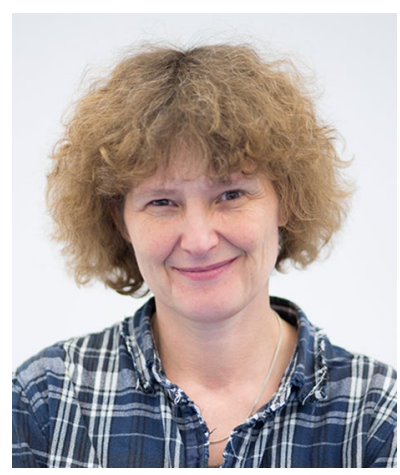

E. J.Milner-Gulland is Tasso Leventis Professor of Biodiversity at the University of Oxford. Her research includes developing and applying methods for understanding, predicting, and influencing human behaviour. She aims to ensure that all her research addresses issues identified by practitioners and is carried out collaboratively with end-users.

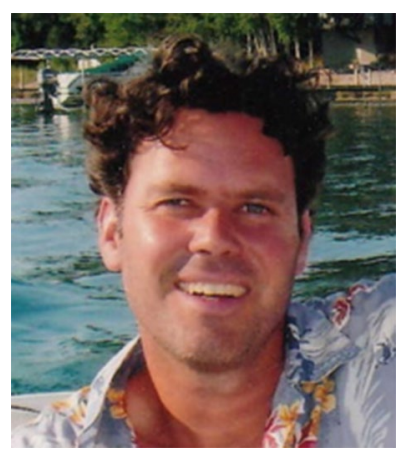

Prof. Myron A. Peck is a biological oceanographer at the University of Hamburg Institute of Marine Ecosystem and Fisheries Science (IFM) and Center for Earth System Research and Sustainability (CEN). His research combines field surveys, laboratory experiments and modelling to explore how climate change and other drivers influence marine ecosystems.

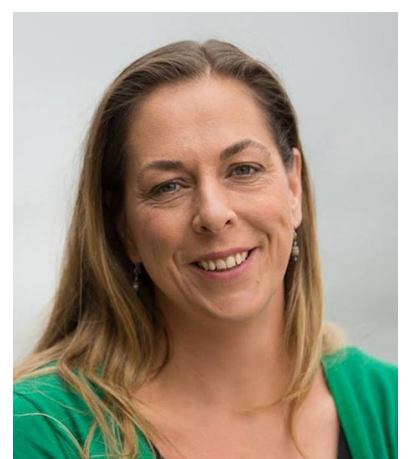

Professor Gretta T. Pecl is an Australian Research Council Future Fellow at the Institute for Marine and Antarctic Studies at the University of Tasmania, and the Director of the Centre for Marine Socioecology. Her research interests include ecological implications of marine climate change, human adaptation to these changes, citizen science and science communication.

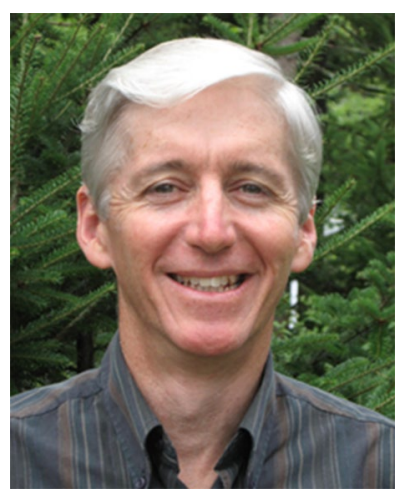

Dr. Robert L. Stephenson is a research scientist with the Canadian Department of Fisheries and Oceans and former Director of the St. Andrews Biological Station. From 2012 to 2017 he was Principal Investigator of the Canadian Fisheries Research Network-an NSERC-funded network that linked academics, industry and government in collaborative fisheries research across Canada.

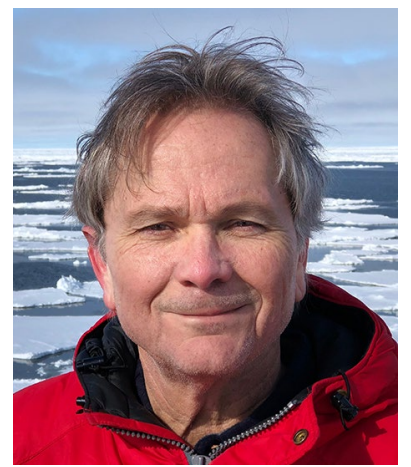

Francisco Werner is the Chief Science Advisor of U.S. NOAA's National Marine Fisheries Service. He leads efforts to provide the science needed to support sustainable fisheries, protected species and ecosystems. Cisco's research focuses on the effects of physical forcing on the structure and function of marine ecosystems. 